Products and Services In Practice is provided to readers using text and images from the manufacturer, supplier or distributor and does not imply endorsement by $B D J$ In Practice. Normal and prudent research should be exercised before purchase or use of any product mentioned.

Please send product and services news through to David Westgarth, BDJ In Practice via: David.Westgarth@bda.org

\section{Changing smiles, discreetly}

You may be experiencing more enquiries about orthodontics, as patients want to invest in improving their smile.

With the Incognito Appliance System from 3M Oral Care, your patients won't have to feel self-conscious about wearing an appliance, because it is fitted behind the teeth.

It will be customised to the oral cavity and will gently move their teeth into the desired position. The results will be predictable, enduring and even lifechanging, offering an ideal solution for a range of patients.

With the Incognito Appliance System, they can go about their daily lives, knowing that they are on the path to achieving the smile of their dreams.

To find out how you can offer this system at your practice, contact the $3 \mathrm{M}$ team on 08458734066 or visit http:// solutions.3m.co.uk/wps/portal/3M/ en_GB/orthodontics_EU/Unitek//

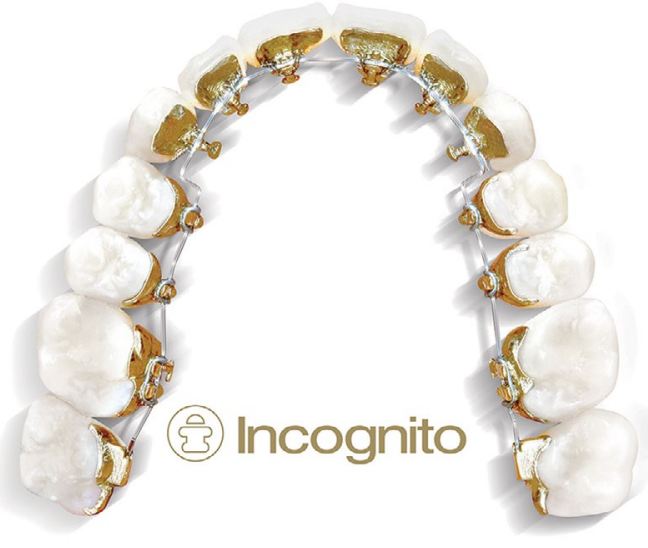

\title{
Access endo success
}

Precision-built and created in the UK, EndoPerfection's VaryFlex files offer amazing value without compromising on quality and are simple to use.

For example, the files have incredible flexibility due to the heat-treated nickel titanium alloy and a $11 \mathrm{~mm}$ shank, which is shorter than usual, making working with VaryFlex very comfortable and easy.

They can be used in much the same way as some other, popular files out there,

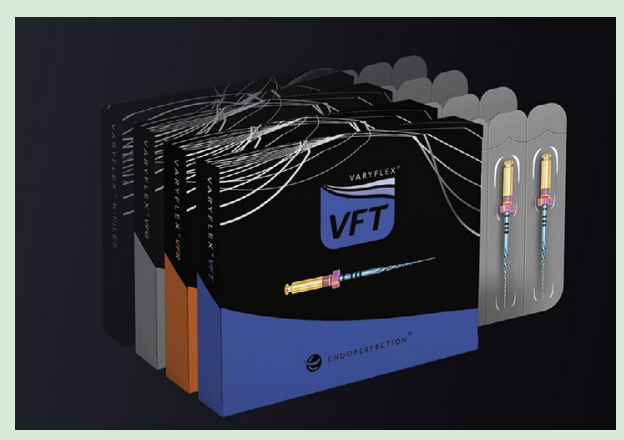

however file creator, EndoPerfection, believe very much in the importance of ongoing training and supporting clinicians in their endeavours.

So, following the success of the online training in the last year, there are now also opportunities to enjoy this form of training.

Alongside movies demonstrating quick and easy tips for rubber dam placement and using VaryFlex Reciprocating and Taper files, there are virtual training events using conference call technology. Products are delivered to delegates in advance, so they get a great experience without having to travel.

VaryFlex is available exclusively from Wrights at www.wright-cottrell. co.uk/new-endoperfection-filesrange/. Information on training courses can be found at https:// endoperfection.com/products/ hands-on-rotary-endo-training.

\section{Wipe away your infection control fears}

CaviWipes from Kerr are all-in-one, ready-to use disinfectant wipes presaturated with CaviCide solution for the effective removal of dirt as well as quick and broad-spectrum disinfection.

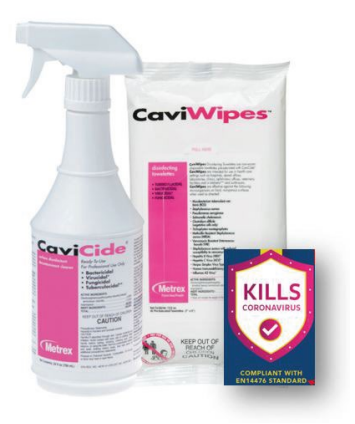

Available

in a flat

pack or

cannister for

convenience,

CaviWipes

have a low

alcohol

content

(17\%),

preventing bioburden from becoming insoluble and stubbornly adherent to surfaces, for a more effective clean and disinfection. They do not contain aldehydes, phenols, bleach or other toxic chemicals.

CaviCide is effective against Human Coronavirus and with these extra-large and thick wipes, fungicidal, bactericidal, virucidal and tuberculocidal disinfection is achieved in just 3 minutes.

In addition, the non-woven fabric allows the tissue to remain intact, without bunching up or drying out during use.

For further information, visit www. kerrdental.com/en-uk or contact your local rep. 